\title{
Neues aus Neuropsychologie und Psychotherapie
}

Neuropsychologie und Psychotherapie sind Bereiche der Klinischen Psychologie, die in den vergangenen Jahren für die Psychiatrie stark an Bedeutung und an eigenständigem Profil gewonnen haben. Nach längerer Zeit sind diese Disziplinen wieder aus dem Schatten von Pharmakologie und Neurobiologie herausgetreten. Im Bereich der Diagnostik hängt dies mit der zunehmenden Aufmerksamkeit zusammen, die in den vergangenen Jahren die Demenzerkrankungen, insbesondere die Alzheimersche Krankheit, gefunden haben. Hier stellen die Früherkennung der Erkrankung sowie die Abbildung der Wirksamkeit krankheitsmodifizierender Therapiemethoden die Neuropsychologie vor neue Herausforderungen. Auf dem Gebiet der Schizophrenien ist die entscheidende Bedeutung von Negativsymptomen und kognitiven Störungen für den Langzeitverlauf lange bekannt. Die Bedeutung der Neuropsychologie für die Diagnostik und die der Psychotherapie für die Therapie dieser Erkrankung ist allerdings noch nicht allgemein anerkannt.

Neuropsychologische Einschränkungen psychiatrischer Patienten erschließen sich häufig nur teilweise in der psychopathologischen Untersuchung. Ihre Erfassung bedarf spezifischer Verfahren und qualifizierter Untersucher, die in der Anwendung und Ergebnisinterpretation für diese Instrumente geschult und geübt sind. Vielfalt und Stellenwert neuropsychologischer Untersuchungsverfahren werden in dem einführenden Übersichtsartikel von Blessing et al. dargestellt. In dem folgenden Beitrag werden die wesentlichen neuropsychologischen Einschränkungen von an Schizophrenie erkrankten Patienten und die Bedeutung dieser Einschränkungen für Funktionsniveau und Langzeitverlauf dargestellt.
Auf dem Gebiet der Psychotherapie erscheint bemerkenswert, dass in den vergangenen Jahren in zunehmendem Ausmaß auch Krankheitsbilder psychotherapeutisch behandelt werden, bei denen man dies noch vor einigen Jahren als wenig hilfreich, vielleicht sogar als schädlich angesehen hat. In dem Beitrag von Veselinović et al. wird der aktuelle Stand in der Anwendung der Dialektisch-Behavioralen Therapie bei Patienten mit Borderline-Persönlichkeitsstörung dargestellt, ein Verfahren, das sich in dieser Indikation schon seit einigen Jahren bewährt hat. Sinnvoll und hilfreich kann die Anwendung verhaltenstherapeutischer Techniken aber auch bei an Schizophrenie erkrankten Patienten mit therapierefraktärer Positivsymptomatik sein. Diese interessante, mitunter kontrovers diskutierte Thematik wird in dem Beitrag von Vauth dargestellt. Möglicherweise noch umstrittener ist die Durchführung kognitiver Trainingsmaßnahmen bei an Demenz erkrankten Patienten. Nach der Ära des Realitäts-Orientierungs-Trainings (ROT), das in der Vergangenheit mitunter wenig einfühlsam durchgeführt wurde, stehen derzeit bei der Psycho-, Sozio- und Ergotherapie Verfahren im Vordergrund, die sich primär am Wohlbefinden der Patienten orientieren, z.B. die Validation. Das Potenzial und die Durchführbarkeit kognitiver Trainingsmaßnahmen sind noch weitgehend unbekannt. Meine Arbeitsgruppe hat sich dieses Themas angenommen; erste Untersuchungsergebnisse zur Auswirkung eines Gedächtnistrainings auf Lebensqualität und Gedächtnisleistung von an Demenz erkrankten Patienten sind in dem Beitrag von Schimmel et al. dargestellt.

Ich hoffe, die Beiträge dieses Heftes finden Ihr Interesse. Sie sind meiner Meinung nach informativ und anregend. Ich wünsche Ihnen viel Vergnügen bei der Lektüre!

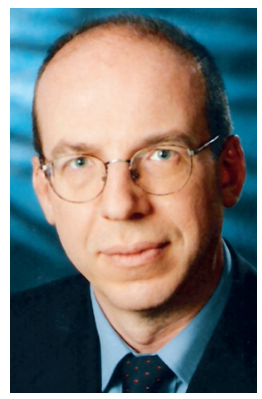

Prof. Dr. Georg Adler 